\title{
New pulsation pattern of RZ Cas observed spectroscopically in 2006
}

\author{
Holger Lehmann, ${ }^{1}$ David Mkrtichian ${ }^{2}$ \\ 1 Thüringer Landessternwarte, D-7778 Tautenburg, Germany \\ ${ }^{2}$ ARCSEC, Sejong University, Seoul 143-747, Korea
}

\begin{abstract}
We investigated the radial velocities of the mass-accreting Algol-type star RZ Cas measured from new observations obtained in 2006. After subtracting an improved orbital solution and all low-frequency variations we searched for the signature of short-term non-radial pulsations. We found three pulsation modes where one was never observed before. Also the amplitudes of the two other modes have changed since 2001. During the eclipse phases we observed a much stronger increase of the pulsation amplitudes than in 2001 and a less pronounced anomaly of the Rossiter-McLaughlin effect.
\end{abstract}

RZ Cas is a member of the mass-accreting Algol-type stars with pulsating components (oEA stars, Mkrtichian et al. 2006). Multi-periodic, $\delta$ Scuti-type non-radial pulsations (NRPs) were observed for the primary, from photometry (Rodríguez et al. 2004) and from spectroscopy (Lehmann \& Mkrtichian 2004, Paper I). The pulsations possess timely changing amplitude and frequency patterns as well as orbital amplitude modulation. RZ Cas shows a very pronounced Rossiter-McLaughlin effect (RME). The RME is a distortion in the orbital radial velocity (RV) curve that can be observed during the eclipse phases of eclipsing binaries with rapidly rotating components (during the eclipse the symmetry of the rotational broadening of the spectral lines is lifted in a time dependent manner). In RZ Cas the RME is anomalous, i.e. the positive and negative deviations from the orbital curve are of different strengths.

To study the changes in the oscillation spectrum and the effects of the circum-primary accretion envelope on the RME and on the amplitude modulation of NRP modes further, we continued to monitor the star spectroscopically. We obtained 498 spectra in 7 nights in 2006 with the Coude-Echelle spectrograph at the 2-m telescope of the Thüringer Landessternwarte Tautenburg with a spectral resolution of 30000 and typical S/N of 80 . Based on the new RVs measured by a cross-correlation technique and on the data from 2001 (Paper I) we improved the orbital solution. In the residuals we observed low-frequency trends within single runs. After removal of the calculated orbital RVs and of the low-frequency trends (using spline fits in the second case) we investigated the star for NRPs in the high-frequency domain.

Rodríguez et al. (2004) found one frequency of $64.1935 \mathrm{c} / \mathrm{d}$ in their photometric data from 1999. In the time between 1999 and 2001 this mono-periodicity changed multi-periodicity. Table 1 compares the frequencies obtained from our new data with those from 2001 ( $\mathrm{Pa}$ per I). RZ Cas has changed its pulsation pattern again. For the first time we observe three pulsation frequencies; $f_{3}$ was never observed before (correctly speaking, also the values of $f_{2}$

Table 1: High-frequency variations found in the radial velocities of RZ Cas. Errors were derived by the PERIOD04 code (Lenz \& Breger 2005) and given in parentheses, in units of the last digit(s).

\begin{tabular}{|c|cc|crc|}
\hline & \multicolumn{2}{|c|}{2001} & & 2006 & \\
& $\mathrm{f}[\mathrm{c} / \mathrm{d}]$ & $\mathrm{A}[\mathrm{m} / \mathrm{s}]$ & $\mathrm{f}[\mathrm{c} / \mathrm{d}]$ & $\mathrm{A}[\mathrm{m} / \mathrm{s}]$ & phase \\
\hline$f_{1}$ & $64.189(6)$ & $99(26)$ & $64.2703(3)$ & $203(14)$ & $0.78(1)$ \\
$f_{2}$ & $56.600(4)$ & $151(27)$ & $55.7610(6)$ & $94(14)$ & $0.54(2)$ \\
$f_{3}$ & - & - & $62.4062(4)$ & $145(14)$ & $0.85(2)$ \\
\hline
\end{tabular}


are significantly different and represent two different frequencies). For the determination of amplitude variations we built overlapping phase bins from all runs in 2006, each bin of width 0.2 in orbital phase; 20 such phase bins covered the orbital period in steps of 0.05 . Then we applied sinusoidal fits including the three frequencies to the data of each bin. Fig. 1 shows the results. All three frequencies show small amplitude modulations in the out-of-eclipse phases and a strong (3-4 times) increase of amplitude during eclipse. This increase is much stronger than observed in 2001 (Paper I). Comparing the orbital solutions obtained from the 2001 data (Paper I) and from the new data alone we can confirm the increase of the period of about 2 sec since 2001 as obtained from photometry (Mkrtichian et al. 2007). The anomaly of the RME is less pronounced in the 2006 data compared to 2001 but still present.

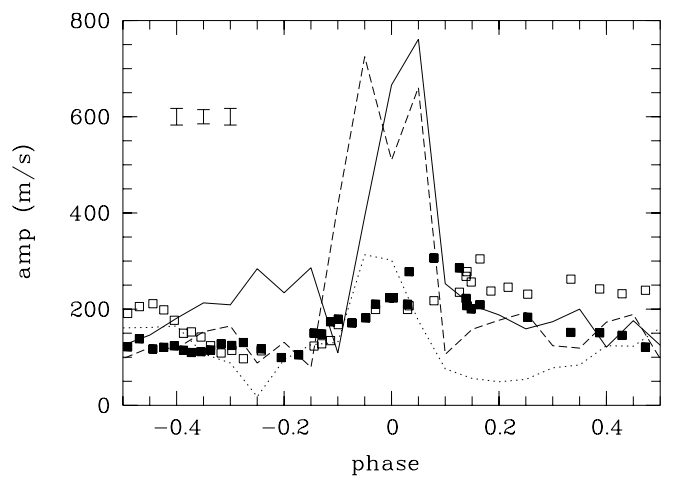

Figure 1: Amplitudes of the RV variation for frequencies $f_{1}$ (solid), $f_{2}$ (dotted), and $f_{3}$ (dashed). Mean errors are indicated by the error bars, from left to right for $f_{1}$ to $f_{3}$. Squares show the variation observed in 2001 (Paper I), filled squares correspond to $f_{1}$, open squares to $f_{2}$. Phase zero means the epoch of minimum light.

Our conclusion so far is that all variations with the orbital period obtained from the 2001 and from the 2006 data reflect the respective density distributions of circum-stellar disk-like gas structures. In both cases the density and its gradient are highest near the epoch of minimum light causing the large amplitudes of RV variations at these phases and to the asymmetry of the RME. Besides more or less steady changes in the circum-stellar density distribution we assume also transient phases of abrupt, massive changes. The first give rise to the different low-frequency trends in RV observed for the same rotation phases with time. The second are assumed to originate from high mass-transfer episodes that change the pattern of NRP modes excited in RZ Cas and cause the observed unsteadiness of the rotation period. The assumption of a timely varying attenuation effect of circum-stellar disk-like structures also explains the different shape of the anomalous RME observed in 2001 and 2006.

Results will be discussed in much more detail in a forthcoming paper where we also want to try to model the observed RME (screening effect by the secondary) for different NRP modes and different circum-stellar density distributions obtained from 3D hydrodynamical models.

\section{References}

Lehmann H., Mkrtichian D. E., 2004, A\&A, 413, 293, Paper I

Lenz P., Breger M., 2005, Comm. Asteroseis., 146, 53

Mkrtichian D. E., Kim S.-L., Kusakin A. V., et al., 2006, Astroph. Space Sci., 304, 169

Mkrtichian D. E., Kim S.-L., Rodríguez E., et al., 2007, ASP Conf. Ser., in press

Rodríguez E., Garcia J. M., Mkrtichian D. E., et al., 2004, MNRAS, 347, 1317 